\title{
Modal synthesis of precision control systems
}

\author{
E M Vasiljev ${ }^{1,}{ }^{*}, E A$ Serdechnaya $^{1}, A V$ Tavolzhanskij $^{1}$ \\ ${ }^{1}$ Voronezh State Technical University, 14, Moscow Av., Voronezh, 394026, Russia
}

\begin{abstract}
The problem of synthesis of precision modal control systems is considered. It is noted that a common approach to solving this problem is to consistently meet the requirements for the nature of the transient process and for the indicators of its accuracy. This approach to synthesis is faced with the need to make design decisions under incomplete conditions. In practice, this circumstance leads to obtaining synthesis results with undesirable deviations from technical requirements. When designing precision control systems, such deviations are unacceptable. To eliminate the difficulties that arise, a transition to interval methods for formulating and solving modal synthesis problems is proposed. The theoretical possibility of the interval approach is based on the excessive variety of possible placement of eigenvalues in the spectrum of the characteristic matrix of the system. An example of an interval synthesis of a system with a modal controller and additional output feedback is considered. The restrictions on the spectrum of the specified matrix are formed, which determine the fulfillment of the requirements for the monotonicity of the transient process, the regulation time and the accuracy of the response to harmonic influences. It is noted that the variety of solutions obtained creates the preconditions for a multi-alternative approach to modal synthesis of systems.
\end{abstract}

\section{Introduction}

The principle of modal control, due to the most complete reflection of the cybernetic essence of state feedback, is widely used to control lightly damped, structurally unstable and nonstationary objects [1-10].

The problem of forming the spectrum of eigenvalues of the characteristic matrix arising during the synthesis of such systems is traditionally solved without taking into account the requirements for the accuracy indicators of the response to the reference input signals. These indicators are ensured at the subsequent stages of system design by introducing an external signal model and additional correcting filters into its structure [11-18].

Such a sequential approach to the synthesis of a control system is based on the widespread method of decomposition of the general design problem, and is convenient in methodological terms. However, the separate provision of the requirements for the time

\footnotetext{
* Corresponding author: vgtu-aits@yandex.ru
} 
indicators of the transient process and the indicators of the system accuracy leads to their incompatibility. The most frequent manifestation of this incompatibility is the occurrence of unacceptable oscillability of the transient process during stepwise effects on the system. Eliminating oscillability due to the deterioration of the accuracy of the system comes down to finding a compromise. It is undesirable in the general case and impossible when synthesizing high-accuracy systems.

In this paper, a method for the synthesis of modal control systems is presented, which guarantees the joint provision of the required quality of the transient process and the accuracy of the system.

Synthesis procedures will be considered using two typical structures as an example:

- structure with output feedback;

- structure with an additional input channel for the reference input signal.

\section{Synthesis of control in a structure with output feedback}

\subsection{Synthesis method}

Let a completely controllable and, in the general case, unstable object be known:

$$
\begin{aligned}
& \dot{x}=B x+N u ; \\
& y=A x,
\end{aligned}
$$

for which the following are defined: $B$ - characteristic matrix, $N$ - control matrix, $A$ output matrix, $x$ - state coordinate vector, $u$ - control, $y$ - controlled variable.

For this object, we set the task of synthesizing a modal control system with the following quality indicators:

- monotonous nature of the transition process;

- regulation time $t_{r}$;

- zero steady-state error for the constant reference input signal $g(t)=$ const, i.e. first order astatism with respect to the input signal;

- the maximum allowable error $\varepsilon_{v}$ for the velocity action $g(t)=v_{\max } t$, i.e. quality factor $D_{v}=v_{\max } / \varepsilon_{v}$ in speed;

- maximum permissible relative errors $\delta_{1}$ and $\delta_{2}$ of reproduction of the amplitude of harmonic influences at frequencies $\omega_{1, g}$ and $\omega_{2, g}$.

To solve this problem, a system is formed with the structure shown in figure 1.

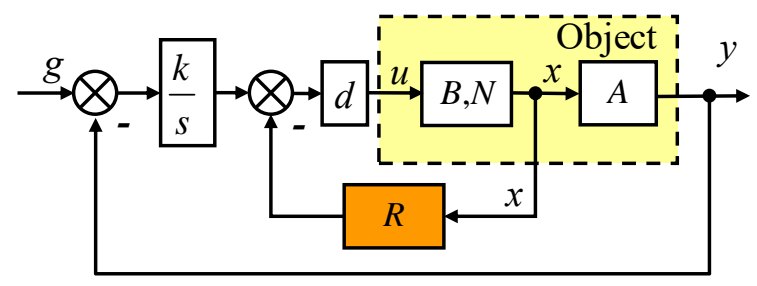

Fig. 1. Output feedback system.

Where: $R=\left[\begin{array}{llll}r_{1} & r_{2} & \ldots & r_{n}\end{array}\right]-$ modal regulator; $d$ and $k / s-$ additional inertialess and integrating links introduced into the system to ensure the above quality indicators $[19,20]$.

For the structure in figure 1, let us find the equation of motion in the Laplace images: 


$$
y(s)=A \cdot Q^{-1} \cdot N \cdot d \cdot \frac{k}{s} \cdot g(s) ; \quad Q=s \cdot E-B+N \cdot d \cdot R+N \cdot d \cdot A \cdot \frac{k}{s} .
$$

The characteristic polynomial of the system is obtained from (2):

$$
|Q|=s^{n+1}+\left(a_{n-1}+d \cdot r_{n}\right) \cdot s^{n}+\left(a_{0}+d \cdot r_{1}\right) \cdot s+k \cdot d .
$$

The transfer function of the closed system takes the form:

$$
W(s)=\frac{k \cdot d}{s^{n+1}+\ldots+\left(a_{0}+d \cdot r_{1}\right) \cdot s+k \cdot d},
$$

from which it directly follows that the system has the property of astatism, i.e. already at the structural level, for any parameters of the system, a zero steady-state error is ensured for constant actions $g(t)=$ const [21].

Since the roots of polynomial (3) are functions of free parameters of the system $s_{1}\left(r_{1}, \ldots, r_{n}, k, d\right), \ldots, s_{\mathrm{n}+1}\left(r_{1}, \ldots, r_{n}, k, d\right)$, and the number of variables $(n+2)$ is greater than number of roots $(n+1)$, then it becomes possible not only to place the roots of the polynomial in the desired way ( $n+1$ equation), but also to provide the required quality factor $D_{v}$ in speed:

$$
D_{v}=\frac{k \cdot d}{a_{0}+d \cdot r_{1}}
$$

A system of $n+2$ equations with $n+2$ unknowns will be obtained. In this case, the relative errors $\delta_{1}$ and $\delta_{2}$ of the reproduction of harmonic influences remain undefined.

To increase the number of free variables, as well as reduce the computational complexity of solving the resulting multidimensional problem, we use two methods:

- we pass from equations to interval conditions, i.e. compose a system of inequalities that reflect the requirements for the accuracy of the system and the nature of the transient process;

- we restrict ourselves to two-sided interval specification of only a part of the roots of the characteristic polynomial that have a dominant value and make the remaining roots insignificant, moving them away from the dominant ones using one-sided inequalities.

As a result, we get the following problem:

$$
\left\{\begin{array}{l}
-\alpha \cdot \Omega \leq s_{1}\left(r_{1}, \ldots r_{n}, k, d\right) \leq-\Omega \\
\ldots \\
-\alpha \cdot \Omega \leq s_{m}\left(r_{1}, \ldots r_{n}, k, d\right) \leq-\Omega \\
s_{m+1}\left(r_{1}, \ldots r_{n}, k, d\right) \leq-\beta \Omega \\
\ldots \\
s_{n+1}\left(r_{1}, \ldots r_{n}, k, d\right) \leq-\beta \Omega \\
k \cdot d \geq D_{v}\left(a_{0}+d \cdot r_{1}\right) \\
1-W\left(\omega_{z, g}\right) \leq \delta_{z}
\end{array}\right.
$$


where: $\Omega$ - the desired geometric mean root determined by the given control time; $\alpha$ coefficient that determines the length of the interval; $\beta$ - coefficient specifying the degree of removal of insignificant roots from dominant ones, $\beta>\alpha ; W\left(\omega_{z, g}\right)-$ the modulus of the frequency function $W(j \omega)$ at the frequency $\omega_{z, g} ; z$ - the ordinal number of the harmonic influence.

Thus, the solution of the problem of ensuring the accuracy of harmonic and monotonic control actions with the simultaneous fulfillment of the requirements for the nature of the transient process and the regulation time was reduced to the fulfillment of a system of restrictions (6).

\subsection{Method verification and discussion of results}

Let's consider an example of solving problem (6).

Unstable object is set:

$$
B=\left\lfloor\begin{array}{cc}
0 & 1 \\
0.2 & -3
\end{array}\right\rfloor ; \quad N=\left\lfloor\begin{array}{l}
0 \\
1
\end{array}\right\rfloor ; \quad A=\left[\begin{array}{ll}
1 & 0
\end{array}\right] .
$$

Let us synthesize a system with a monotonic character of the transient process and a regulation time $t_{r}=0.05 \mathrm{~s}$.

For $g(t)=$ const - it is necessary to provide first-order astatism with a speed quality factor $D_{v} \geq 30 \mathrm{~s}^{-1}$.

The harmonic signal $g(t)=0.5 \sin (3 t)$ needs to be reproduced with a static error $\delta_{1} \leq 0.01$, and the signal $g(t)=0.5 \sin (30 t)-$ with a static error $\delta_{2} \leq 0.1$.

For two dominant roots and the regulation time $t_{r}$, we define the geometric mean root $\Omega$ :

$$
\Omega=\frac{T}{t_{r}}=\frac{4.8}{0.05}=96 \mathrm{~s}^{-1},
$$

where: $T$ - constant depending on the order of the desired polynomial. Let's take $\Omega=100 \mathrm{~s}^{-1}$ and choose $\alpha=1.2 ; \beta=5$.

For the given conditions, solution (6) is obtained: $R=\left[\begin{array}{ll}640 & 3.2\end{array}\right] ; k=33000 ; d=990$.

Roots of the characteristic polynomial: $s_{1}=-101 ; s_{2}=-110 ; s_{3}=-2960$.

The results of modeling the synthesized system are tabulated.

Table 1. Comparison of the required and actual quality indicators of the synthesized system.

\begin{tabular}{lcccc}
\hline \multirow{2}{*}{ Value } & \multicolumn{4}{c}{ Quality indicator } \\
\cline { 2 - 5 } & $\mathrm{t}_{r}, \mathrm{c}$ & $\delta_{1}$ & $\delta_{2}$ & $D_{v}, \mathrm{~s}^{-1}$ \\
\hline Required & 0.05 & 0.01 & 0.1 & 30 \\
Actual & 0.045 & 0.0008 & 0.08 & 52 \\
\hline
\end{tabular}

Transient processes in the system are shown in figure 2 and figure 3. 


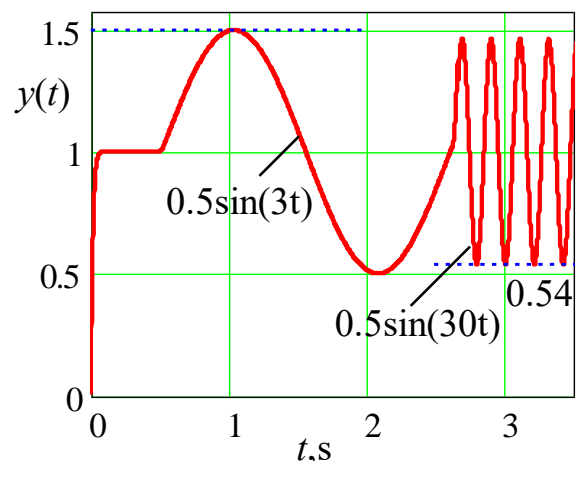

Fig. 2. Transient processes in the system.

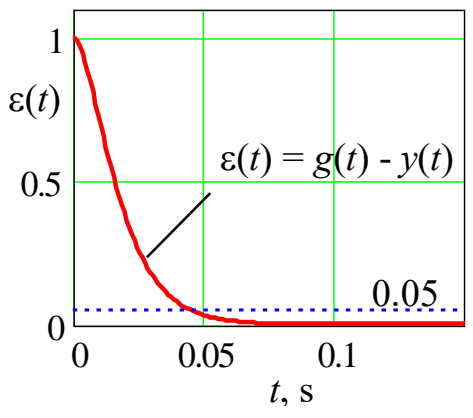

Fig. 3. Change of error in time at $\mathrm{g}(\mathrm{t})=1$.

The requirements for the quality indicators of the transient process and for the accuracy of the response to monotonic and harmonic influences are met.

It should be noted that the use of interval conditions of the synthesis problem makes it possible to obtain several feasible solutions depending on the initial point of the search. This circumstance determines the possibility of the subsequent choice of one of the obtained solutions according to additional criteria that are not taken into account in the formal setting of the problem. In the general case, this possibility allows proceeding to the formulation of a multi-alternative synthesis problem [22-26].

\section{Synthesis of control in a structure with an additional reference input channel}

\subsection{Synthesis method}

Let's move on to considering a more perfect structure of the control system, which allows providing high-order astatism in it, i.e. zero error not only for constant, but also for velocity actions of the form $g(t)=g_{0}+v \cdot t$, figure 4 .

In this structure, the partial invariance of the system error (up to the second derivative of the reference input signal $g(t)$ ) is realized by choosing the corresponding values of the parameters $c_{0}$ and $c_{1}$ of the additional reference input channel. 
To analyze the dynamic properties of the system shown in figure 4, let's consider an object with a differential equation containing the derivatives of the control action on the right side:

$$
y^{(n)}+\ldots+a_{1} \dot{y}+a_{0} y=b_{m} u^{(m)}+\ldots+b_{1} \dot{u}+b_{0} u,
$$

where $a_{0}, \ldots, a_{n-1}, b_{0}, \ldots, b_{m}-$ constant coefficients.

As a result of constructing a modal controller $R=\left[\begin{array}{lll}r_{1} & r_{2} & \ldots\end{array} r_{n}\right]$, the equation of motion of the closed part of the system will take the form:

$$
y^{(n)}+\ldots+a_{1, M} \dot{y}+a_{0, M} y=b_{m} u^{(m)}+\ldots+b_{0} u,
$$

in which the coefficients $a_{0, \mathrm{M}}, \ldots, a_{\mathrm{n}-1, \mathrm{M}}$ determine the desired location of $n$ roots of the characteristic polynomial - the poles of the system. In this case, the polynomial of the numerator of the transfer function of the closed-loop system contains roots (zeros of the system) that can dominate over its poles.

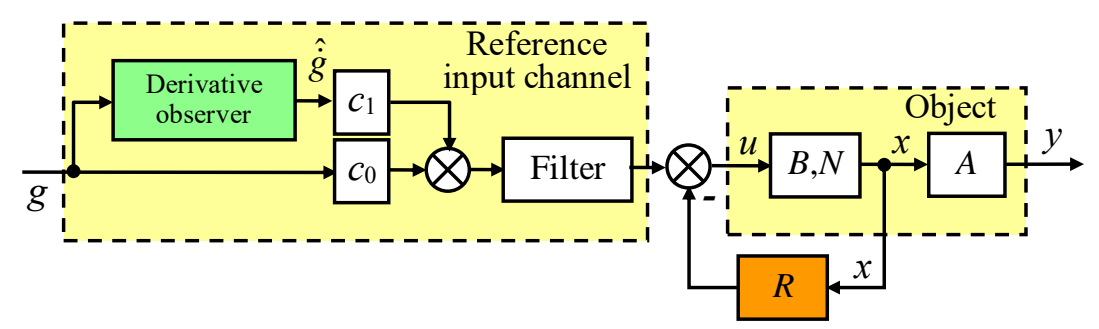

Fig. 4. The structure of the system with a differentiating observer of the reference input.

In the general case, we can assume that some part of the poles (set $S_{1}$ ) forms the required character of the transient process and regulation time, and the remaining $\left|S_{2}\right|$ free roots $\left(\left|S_{2}\right|=n\right.$ - $\left.\left|S_{1}\right|\right)$ can be located at a sufficient distance from $S_{1}$ so that the roots of $S_{2}$ do not significantly affect the quality of the transient process.

We now introduce the observer of the first derivative of the reference input signal at the input of the system, see figure 4.

For the resulting complete structure, a factor $c_{1} s+c_{0}$ appears on the right-hand side of equation (10), which coefficients $c_{0}$ and $c_{1}$ (in the case of compensation of the object's zeros) must satisfy the condition of the second-order astatism $c_{1} s+c_{0}=a_{1, \mathrm{M}} s+a_{0, \mathrm{M}}$.

The transfer function of the system will take the form:

$$
W(s)=\frac{\left(b_{m} s^{m}+\ldots+b_{1} s+b_{0}\right) \cdot\left(c_{1} s+c_{0}\right)}{s^{n}+\ldots+a_{1, \mathrm{M}} s+a_{0, \mathrm{M}}} .
$$

If the zero $\left(\lambda=-c_{0} / c_{1}\right)$ of the transfer function $W(s)$ turns out to be dominant with respect to the poles $S_{1}$ of the closed-loop system, then during the response to the stepwise input action, oscillability (overshoot) of the output value $y(t)$ will occur.

To eliminate this oscillability, it is necessary to compensate for zero $\left(-c_{0} / c_{1}\right)$, i.e. introduce an additional pole $s_{2} \in S_{2}$ corresponding to the condition $s_{2} \geq\left(-c_{0} / c_{1}\right)$ into the desired polynomial of the system.

In this case, the uncertainty of the system synthesis problem arises: to build a modal controller, it is necessary to know the parameters of the reference channel $c_{0}$ and $c_{1}$, and to build the reference channel, it is necessary to know the characteristic polynomial of the 
closed-loop system: in this example - two lower coefficients $a_{1, \mathrm{M}}$ and $a_{0, \mathrm{M}}$, i.e. to know the parameters $\left[r_{1} r_{2} \ldots r_{n}\right]$ of the controller.

The resolution of this uncertainty requires a preliminary answer to the question about the theoretical possibility of placing at least one root $\theta$ of the polynomial $s^{n}+\ldots+a_{1, \mathrm{M}} s+a_{0, \mathrm{M}}$ in such a way that the inequality $\theta \geq\left(-a_{0, \mathrm{M}} / a_{1, \mathrm{M}}\right)$ holds [27-29].

Restricting ourselves to the case of negative real roots that is important for this problem, we prove the following theorem.

Theorem. For a Hurwitz polynomial $s^{n}+\ldots+a_{1} s+a_{0}$ with a set $S$ of real roots $(|S|=n)$ for an arbitrary root $\theta \in S$, the relation $\theta<\left(-a_{0} / a_{1}\right)$ is valid, i.e. the value $\left(-a_{0} / a_{1}\right)$ is the upper bound for the values of the roots of the polynomial.

Proof. By Vieta's theorem, we have:

$$
\begin{aligned}
& a_{0}=(-1)^{n} s_{1} s_{2} \ldots s_{n} \\
& a_{1}=(-1)^{n-1} \underset{1 \leq i_{1}<i_{2} \ldots<i_{n-1} \leq n}{\sum s_{i_{1}} s_{i_{2}} \ldots s_{i_{n-1}}}
\end{aligned}
$$

Let denote $\theta$ through an arbitrary root $\theta \in S$. Then, to prove the theorem, it is sufficient to establish the inequality:

$$
-\frac{(-1)^{n} s_{1} s_{2} \ldots s_{n}}{(-1)^{n-1}\left[s_{1} s_{2} \ldots s_{n-1}+\ldots+s_{2} s_{3} \ldots s_{n}\right]}>\theta .
$$

Let's rewrite (13) as:

$$
\frac{s_{1} s_{2} \ldots s_{n-1}}{s_{1} s_{2} \ldots s_{n}}+\ldots+\frac{s_{2} s_{3} \ldots s_{n}}{s_{1} s_{2} \ldots s_{n}}<\frac{1}{\theta}
$$

or:

$$
\frac{1}{s_{n}}+\frac{1}{s_{n-1}}+\ldots+\frac{1}{\theta}+\ldots+\frac{1}{s_{1}}<\frac{1}{\theta} .
$$

Since all roots are negative, inequality (14) holds for all roots of the polynomial under consideration, i.e. the value $\left(-a_{0} / a_{1}\right)$ is their upper limit.

The theorem is proved.

Corollary. The theoretical inaccessibility of the condition of full compensation $\theta \geq(-$ $\left.a_{0, \mathrm{M}} / a_{1, \mathrm{M}}\right)$, or, equivalently, $s_{2} \geq\left(-\mathrm{c}_{0} / c_{1}\right)$, nevertheless leaves the practical possibility of an arbitrarily close approximation to its fulfillment.

This possibility is indicated by inequality (14), from which it follows that choosing the root $s_{2}=\theta$ dominant (maximum) in the desired characteristic polynomial and placing all other roots $s_{i} \neq s_{2},(i=1, . ., n-1)$ in accordance with the condition $s_{i} \ll s_{2}$, one can come to the relation:

$$
\frac{1}{s_{2}}+\varepsilon<\frac{1}{s_{2}}
$$


where the quantity $\varepsilon$ determined by the condition $s_{i} \ll s_{2}$ can be chosen arbitrarily small. The tendency of inequality (15) to equality will mean the approach to the fulfillment of the compensation condition $s_{2}=\left(-a_{0, \mathrm{M}} / a_{1, \mathrm{M}}\right)$.

Thus, on the basis of the above interval analysis, we can propose the following synthesis technique for a modal control system, performed according to the structure in figure 4:

1) one or a group of multiple roots $S_{1}$ is determined based on the specified control times (see (8));

2) the root $s_{2}$ with a dominance coefficient, for example, $\beta>100$, is determined with respect to the roots $S_{1}$;

3) the remaining free roots are made insignificant by placing them at a sufficient distance to the left of the roots $S_{1}$ and $S_{2}$;

4) a modal regulator, which forms the desired characteristic polynomial $s^{n}+\ldots+a_{1, \mathrm{M}} s+a_{0, \mathrm{M}}$ of the system, is calculated based on the location of the roots given in this manner;

5) the values $c_{0}=a_{0, \mathrm{M}}$ and $c_{1}=a_{1, \mathrm{M}}$ of the reference input observer are assigned;

6) a filter (correcting link) $W_{k}(s)$ of the following form is introduced into the reference channel:

$$
W_{k}(s)=\frac{1}{b_{m} s^{m}+\ldots+b_{1} s+b_{0}},
$$

which eliminates the influence on the characteristics of the system of zeros of the object's transfer function.

\subsection{Method verification and discussion of results}

Let us demonstrate the efficiency of the method using a numerical example.

Consider an object given in the form (1) by matrices $B, N, A$ :

$$
\begin{aligned}
B & =\left[\begin{array}{ccccc}
-4 & 4 & 8 & 0 & 0 \\
25000 & -25000 & -50000 & 1800 & 0 \\
-22 & 22 & 0 & 0 & 0 \\
0 & 200 & 0 & -1600 & 5 \\
0 & 0 & 0 & 0 & -660
\end{array}\right] ; \quad N=\left[\begin{array}{c}
0 \\
0 \\
0 \\
0 \\
26000
\end{array}\right] \\
A & =\left[\begin{array}{lllll}
0.019 & 0 & 0 & 0 & 0
\end{array}\right]
\end{aligned}
$$

The numerator of the transfer function of the object is $17784000 \cdot(s+44)$, i.e. its transfer function contains zero $\lambda=-44$. The roots of the object are in the interval $(-24944 ;-0.036)$.

The system requires a monotonic transient process with a regulation time $t_{r}=0.01 \mathrm{~s}$ and second-order astatism.

From the given $t_{r}$ we determine $s_{1}$ :

$$
s_{1}=-\frac{T}{t_{r}}=-\frac{3}{0,01}=-300 \mathrm{~s}^{-1} .
$$

Let's determine the compensating root $s_{2}$ from the condition $s_{2} \geq 100 s_{1}: s_{2}=-1$. 
For the remaining roots, we assign values that are significantly lower than $s_{1}: s_{3}=s_{4}=-$ $5000 ; s_{5}=-24944$.

The results of calculating the modal controller: $R=\left[\begin{array}{lllll}4503 & 26 & 29791 & 105 & 0.31\end{array}\right]$.

Coefficients of the characteristic polynomial: $a_{0, \mathrm{M}}=1.871 \cdot 10^{14} ; a_{1, \mathrm{M}}=1.878 \cdot 10^{14}$. The ratio of the coefficients: $\left(-a_{0, \mathrm{M}} / a_{1, \mathrm{M}}\right)=-0.996$, as expected, is close to the value $s_{2}=-1$.

The transfer function $W_{k}(s)$ of the correcting link compensates for the zero $\lambda=-44$ of the object:

$$
W_{k}(s)=\frac{1}{17784000 \cdot(s+44)} .
$$

Transient processes in the synthesized system were compared with a system without zero compensation $s_{2}$ and polynomial roots: $s_{1}=s_{2}=s_{3}=-300 ; s_{4}=-1000 ; s_{5}=-24944$. The comparison results are shown in figure 5.

Analysis of figure 5 shows that, despite the absence of complex roots of the characteristic polynomial, a significant overshoot $\sigma=30 \%$ occurs in the system without compensation of the observer's zero. Application of the proposed synthesis method practically eliminates oscillability, reducing overshoot to $\sigma=0.4 \%$.

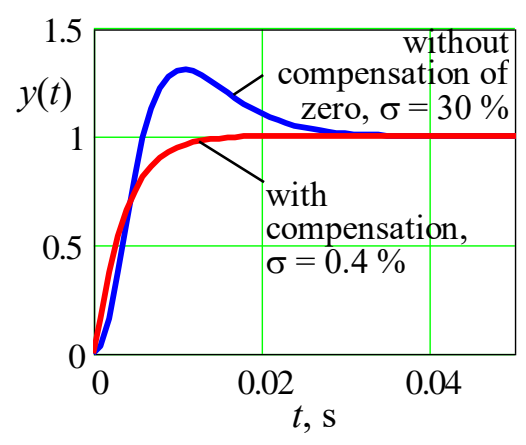

Fig. 5. Comparison of transient processes in systems synthesized with compensation and without compensation of the observer's zero.

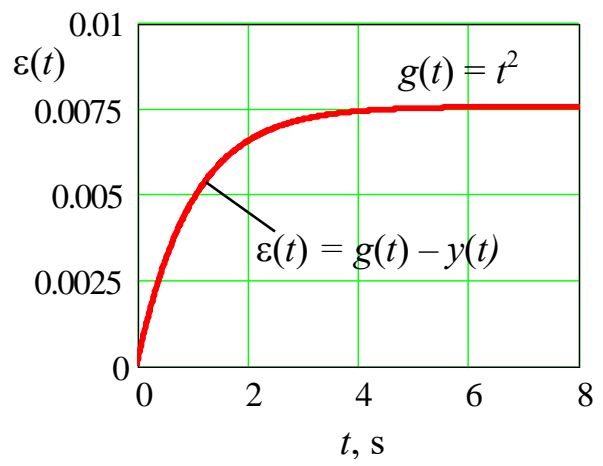

Fig. 6. Checking the steady-state value of the control error when introducing the reference input with constant acceleration. 
The presence of second-order astatism in the system is confirmed by the constant value of the steady-state error $\varepsilon(t)=g(t)-y(t)$ when the input signal is supplied with constant acceleration: $g(t)=t^{2}$, figure 6 .

It follows from figure 6 that the quality factor of the acceleration system is:

$$
D_{a}=\frac{2}{0.0075}=265 \mathrm{~s}^{-2}
$$

Thus, in a system with modal control, the joint fulfillment of the requirements both to the nature of the transient process and to the indicators of its accuracy is ensured without the use of integrators in a closed loop.

\section{Conclusion}

The problem of ensuring the accuracy of modal control systems is traditionally considered as an independent stage of system design, not associated with the stage of calculating the modal controller itself.

The research results presented in the paper show that the indicated approach to modal synthesis of systems leads to problems with incomplete conditions: the solution of each of them requires a preliminary solution to the other.

Ignoring the noted interdependence greatly simplifies the practical synthesis of systems, but at the same time, it becomes necessary to find a compromise between the requirements for the nature of the transient process and the requirements for accuracy indicators. Such a compromise leads to undesirable, and in the case of designing systems with a high astatism order - to unacceptable deviations from the technical specifications.

The proposed method for designing modal control systems eliminates the indicated uncertainty of synthesis subproblems, solving them as a single multidimensional problem of ensuring interrelated quality indicators of the system.

Practical methods of such an approach to synthesis, demonstrated using examples of typical structures with additional output feedback and with a differentiating observer of the reference input, indicate the expediency of formulating and solving the synthesis problem by interval methods. The transition to interval estimates of the feasible search area makes it possible to obtain a guaranteed solution to a multidimensional synthesis problem with a significant reduction in its computational complexity.

\section{References}

1 Dudarenko N A, Slita O V and Ushakov A V 2012 IFAC Proceedings Volumes 45(13) 150

2 Nikolov N, Alexandrova M and Lukov V 2017 15th International Conference on Electrical Machines, Drives and Power Systems (ELMA) Sofia 203

3 Magni J-F 2012 Robust modal control with a toolbox for use with MATLAB (New York: Springer-Verlag New York Inc) p 312

4 Veremey E I, Sotnikova M V, Eremeev V V and Korovkin M V 2014 14th International Conference on Control, Automation and Systems (ICCAS 2014) Seoul 1278

5 Slita O V, Tsventarnyi A Y and Ushakov A V 2012 IFAC Proceedings Volumes 45(13) 15 
6 Belousov E V 2018 IOP Conference Series: Earth and Environmental Science 194 022003

7 Wang L, Liang H R, Mokhlis H, Prokhorov A V and Huat C K 2018 IEEE Industry Applications Society Annual Meeting (IAS) Portland OR 8544580

8 Peukert C, Pöhlmann P, Merx M, Müller J and Ihlenfeldt S 2019 Journal of Machine Engineering 19(2) 32

9 Zhao W and Huang D 2011 Key Engineering Materials 467-469 509

10 Van Gils R W, Speetjens M F M, Zwart H J and Nijmeijer H 2014 International journal of thermal sciences 8234

11 Ryadchikov I, Aranovskiy S, Nikulchev E, Wang J and Sokolov D 2019 IFACPapersOnLine 52(16) 436

12 Stein G L and Konigorski U 2012 IFAC Proceedings Volumes 45(2) 130

13 Bouderbala K, Nouira H, Girault M and Videcoq E 2016 Applied Thermal Engineering 104504

14 Cai K, Tian Y, Wang F, Zhang D and Shirinzadeh B 2017 Robotics and ComputerIntegrated Manufacturing 4477

15 Juqing Y, Dayong W and Weihu Z 2017 Optik 131994

16 Shao X, Liu J and Wang H 2018 Mechanical Systems and Signal Processing 104631

17 Zarei A, Poutari M S and Masoud Barakati S M 2018 ISA Transactions 7499

18 Song C, Lei W and Feng X Y 2011 Procedia Engineering 15573

19 Lapin A V and Zubov N E 2019 Proceedings - 2019 International Russian Automation Conference, RusAutoCon Sochi 8867744

20 Gorkaviy A I, Gorkaviy M A, Melnichenko M A and Solovev D B 2019 International Multi-Conference on Industrial Engineering and Modern Technologies FarEastCon Vladivostok 8934225

21 Dlapa M 2019 IFAC-PapersOnLine 52(11) 134

22 Podvalny S L and Vasiljev E M 2018 MATEC Web of Conferences. 13th International Scientific-Technical Conference on Electromechanics and Robotics “Zavalishin's Readings” St. Petersburg 16102003

23 Podvalny S L and Vasiljev E M 2015 Automation and Remote Control 76(8) 1471

24 Podvalny S L and Vasiljev E M 2015 Automation and Remote Control 76(2) 311

25 Podvalny S L, Vasiljev E M and Barabanov V F 2014 Automation and Remote Control 75(10) 1886

26 Chen T-Y 2014 Information Sciences 286188

27 Ram YM 2010 Proceedings of the Royal Society A: Mathematical, Physical and Engineering Sciences 466(2115) 831

28 Wang X, Wang L and Qiu Z 2014 Applied Mathematical Modelling 38(9-10) 2377

29 Feng X, Wu J and Zhang Y 2018 Applied Mathematical Modelling 64426 